\title{
Erntefaktor in der Wasserkraft - mögliche Schlussfolgerungen für die Bauwirtschaft
}

\author{
Johannes Wall ${ }^{1}$
}

Online publiziert: 12. Mai 2016

(C) The Author(s) 2016. This article is available at SpringerLink with Open Access

\begin{abstract}
The importance of hydropower is increasing. This article presents the environmental impacts of a hydropower plant over its lifecycle in a holistic approach, furthermore its energy payback ratio is calculated. Additionally future aspects and use of environmental product declarations in construction industry are presented.
\end{abstract}

\section{Einleitung/Ausgangssituation}

Die United Nations Sustainable Development Agenda Post2015 sieht im Rahmen ihrer 17 Entwicklungsziele mit der 7. Zielsetzung die Sicherstellung einer leistbaren, zuverlässigen und nachhaltigen sowie modernen Energieversorgung für alle vor. Damit in Verbindung steht das 13. Ziel, von raschen Maßnahmen gegen den Klimawandel und dessen Auswirkungen. In diesem Zusammenhang wird auch der Wasserkraft durch die Energiewende in Deutschland mehr Bedeutung $\mathrm{zu}$ Teil. Durch den Strommarkt $2.0^{1}$ werden neben einem verstärkten Wettbewerb der Erzeugungstechnologien und notwendigen Innovationen im Bereich der Speicherung auch ökologische Aspekte vermehrt in Erscheinung treten. Die Bestrebungen der Forcierung von erneuerbaren Energieträgern spiegeln sich in den Energie- und Klimazielen der Europäischen Union und deren

\footnotetext{
1 Strommarkt 2.0 stellt einen gesetzlichen Ordnungsrahmen für die Versorgungssicherheit, Bezahlbarkeit und Klimaverträglichkeit der Energiewende in Deutschland durch das Strommarktgesetz dar.

Johannes Wall

johannes.wall@tugraz.at

1 Institut für Baubetrieb und Bauwirtschaft, TU Graz, Graz, Österreich
}

Umsetzung durch die einzelnen Mitgliedsstaaten wider (RL2009/28/EG) (RL2000/60/EG). Für Österreich bedeutet dies aufgrund der geografischen Lage u. a. eine Fokussierung auch auf die Wasserkraft. Derzeit werden ca. $55 \%$ der Stromproduktion von Wasserkraftwerken erzeugt. Zufolge der Wasserkraftpotenzialstudie (Pöyry Energy 2008) gibt es ein ausbaufähiges Potenzial von 13 TWh, wovon ein Großteil auf Kleinwasserkraftwerke, also Wasserkraftwerke mit einer Engpassleistung von $<10 \mathrm{MW}$ entfällt. Besonders in der Steiermark gibt es ein Restpotenzial von 1,5-2,5 TWh, welches in den nächsten Jahren realisiert werden könnte und bereits wird.

In dieses thematische Umfeld ordnet sich die gegenständliche ökobilanzielle Betrachtung eines Kleinwasserkraftwerks ein. Anhand eines Laufwasserkraftwerks wurden die Umweltwirkungen über den gesamten Lebensweg von der Errichtung über den Betrieb bis zum Rückbau der Anlage ermittelt (Wall 2011). Ähnliche Untersuchungen wurden bereits von Suwanit und Gheewala (2011) sowie Vega et al. (2013) durchgeführt. Schlussfolgernd aus den Berechnungen der Umweltwirkungen und des Erntefaktors des Kleinwasserkraftwerks werden abschließend Ansätze für die Berücksichtigung umweltbezogener Informationen im Planungs- und Bauprozess präsentiert.

\section{Methodik}

Ein Kleinwasserkraftwerk wurde mit der Methode der Ökobilanzierung gemäß ÖNORM EN ISO 14040 ff (2006) hinsichtlich der potenziellen Umweltwirkungen betrachtet. Im Zuge des 4-stufigen Verfahrens wurde beginnend mit der Zieldefinition sowie der Festlegung des Untersuchungsrahmens eine Sachbilanz erstellt, um die relevanten Stoffströme des zu untersuchenden Systems zu erfassen. 
Abb. 1 Projektgebiet des Kleinwasserkraftwerks (Quelle: Projektunterlagen Energie Steiermark AG)

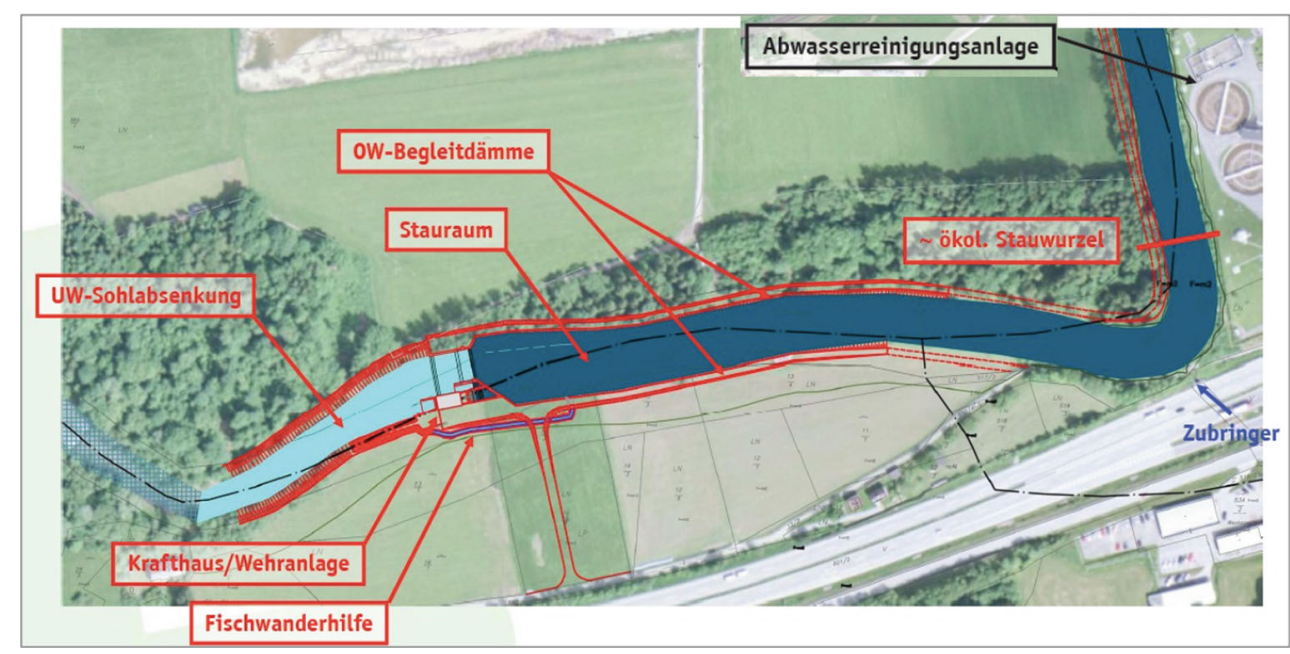

Die Ecoinvent-Datenbank wird zur Erstellung der Sachbilanz genutzt (Ecoinvent 2.2). Die Wirkungsabschätzung erfolgt basierend auf der Sachbilanz anhand der Methode CML 2001 in Microsoft-Excel. Dabei wurden folgende Wirkungsindikatoren ausgewiesen: Versauerungspotenzial (AP), Eutrophierungspotenzial (EP), Treibhauspotenzial (GWP), Stratosphärischer Ozonabbau (ODP), Photooxidantenbildung (POCP), Kumulierter Energieaufwand erneuerbar (renewable energy resources), Kumulierter Energieaufwand nichterneuerbar (non-renewable energy resources).

Die Datengrundlage für das Anwendungsbeispiel stellt ein Kleinwasserkraftwerk der Energie Steiermark dar, mit einer Engpassleistung von ca. $730 \mathrm{~kW}$ (siehe Abb. 1). Als Betrachtungszeitraum (Nutzungsdauer der Anlage) wurden 100 Jahre gewählt. Die funktionale Einheit wurde auf $1 \mathrm{kWh}$ (netto) erzeugter elektrischer Energie des Kleinwasserkraftwerks festgelegt.

Der Sachbilanz zugrunde liegt die Massenaufstellung des Kraftwerks, welches sich aus folgenden Hauptkomponenten zusammensetzt: Baumaßnahmen zur Erschließung des zukünftigen Kraftwerks, Errichtung einer Bauumleitung des Flusses, Herstellung des Krafthauses und der Wehranlage sowie Baumaßnahmen im Oberwasser- und Unterwasserbereich, als auch die Errichtung der Fischwanderhilfe. Des Weiteren wurden auch die Herstellung des Netzanschlusses (Anbindung an das $110 \mathrm{kV}-\mathrm{Netz}$ ) sowie die maschinelle Ausstattung des Kraftwerks und die Komponenten des Stahlwasserbaus (Verschluss und Absperreinrichtungen) mitberücksichtigt. Die Systemgrenzen des Untersuchungsgegenstandes umfassen alle für den Betrieb des Kraftwerks notwendigen Bauteile und Anlagenkomponenten. Diese werden über den gesamten Lebenszyklus des Kraftwerks von der Herstellung über den Betrieb bis zum Rückbau betrachtet. Alle Sachbilanzdaten wurden den Ausschreibungsunterlagen des Projekts entnommen. Im Zuge der Massenermittlung wurden entsprechende Anlagengruppen (Krafthaus, Wehrablage, Oberwasser-, Unterwasserbe- reich, Fischwanderhilfe) und Fertigungsbereiche (Bauumleitung) untersucht, in der darauf folgenden Modellierung wurden die einzelnen Herstellungsprozesse betrachtet und für jede Bauleistung und jedes Bauprodukt die jeweilige Energie- und Stoffbilanz erstellt.

Für eine Abbildung der Nutzung wurde nach 50 Jahren ein vollständiger Austausch der elektromechanischen Ausrüstung und der Stahlwasserbau-Komponenten berücksichtigt. Für den laufenden Betrieb wurde ein Eigenstrombedarf von $2 \%$ der Kraftwerksleistung eingeplant. Der Schmiermittelverbrauch und die notwendigen Instandhaltungs- und Revisionsarbeiten wurden entsprechend der Anlagenstrategie des Betreibers mit einbezogen. Auch das End-of-Life Szenario wurde durch einen vollständigen Rückbau der Anlage mitberücksichtigt und anhand von Entsorgungs- und Recyclingdatensätzen abgebildet. Das Kraftwerk weist mit einer Engpassleistung von ca. $730 \mathrm{~kW}$ und 8640 Betriebsstunden pro Jahr zufolge des verfügbaren Wasserdargebotes ein Regelarbeitsvermögen von 3600 MWh produzierten Stromes auf. Diese Annahmen basieren auf der von der Energie Steiermark durchgeführten Erzeugungsprognose. Alle Sachbilanzdaten wurden unter der Berücksichtigung der Gesamtleistung/Jahr des Kraftwerks und der Nutzungsdauer von 100 Jahren auf die funktionale Einheit bezogen.

Aufbauend auf den Ergebnissen der Ökobilanz wurde der Erntefaktor berechnet. Dieser bezeichnet das Verhältnis der während der Nutzungsdauer erzeugten Energie zu jenen kumulierten Energieaufwendungen, welche für die Herstellung, Betrieb und Rückbau des Kraftwerks aufzubringen sind (Unger und Hurtado 2014; Giesecke et al. 2014). Die Ernte oder der Ertrag einer Stromerzeugungsanlage wird durch die erzeugte elektrische Energie dargestellt. Diese Vorgehensweise ist mit Konzepten des Öko-Controllings oder der ökologischen Rentabilität (Giegrich et al. 2014) vergleichbar. 


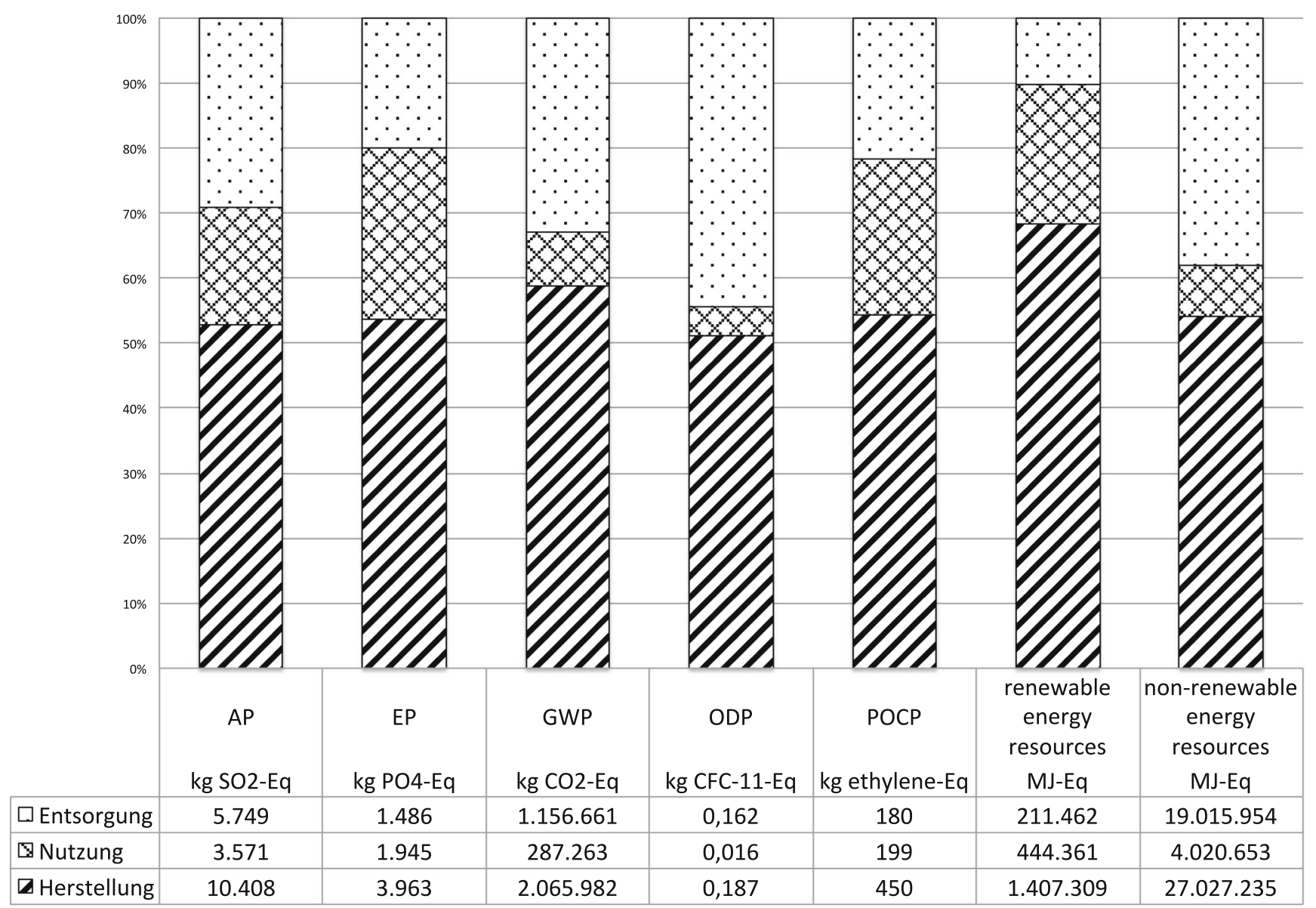

Abb. 2 Umweltwirkungen des Kleinwasserkraftwerks (Quelle: Wall 2011)

\section{Ergebnisse}

Im folgenden Kapitel werden die Umweltwirkungen des Kleinwasserkraftwerks und der berechnete Erntefaktor dargestellt.

\subsection{Umweltwirkungen}

Anhand der Bewertung der Sachbilanzdaten gemäß der Methode CML 2001 wurden Ecoinvent-Datensätze für die Darstellung der Umweltwirkungen über den gesamten Lebenszyklus verwendet. Besonders deutlich ist in Abb. 2 erkennbar, dass die Umweltwirkungen in der Herstellungsphase des Kraftwerks am größten sind, in allen Wirkungskategorien trägt die Herstellungsphase $>50 \%$ zu den Gesamtumweltwirkungen bei. Diese resultieren aus den verwendeten Baustoffen und Transporten im Zuge der Errichtung. Dies zeigt sich auch anhand des Wirkungsindikators ODP, der hauptsächlich auf die Transportketten der Erdarbeiten (Aushub, Rückbau und Renaturierung) zurückzuführen ist. Für den Betrieb des Kraftwerks sind die geringsten Aufwendungen notwendig, da hier keine wesentlichen baulichen Maßnahmen notwendig sind. Die Umweltwirkungen in die- ser Lebenswegphase resultieren aus der Instandhaltung und Instandsetzung der elektromaschinellen Ausrüstung (Austausch der Turbine und des Generators). In der Rückbauund Entsorgungsphase können einige Stoffe (z. B. Metalle) je nach Rückführung in den Wirtschaftskreislauf weiter verwendet werden. Dies wurde auch in den Umweltwirkungen durch die Verwendung von Gutschriften berücksichtigt.

Im Rahmen einer durchgeführten Dominanzanalyse wurden die Umweltwirkungen der benötigten Materialien für die Herstellung der Kraftwerksanlage als maßgeblich identifiziert. Besonders große Umweltwirkungen werden beispielsweise durch die Zementherstellung für den Beton verursacht, sowie die eingesetzten Mengen an Baustahl (Stahlkonstruktionen und Spundbohlen) und den Armierungsstahl. Hohe Umweltwirkungen werden auch durch die Herstellung der elektromaschinellen Ausrüstung wie Turbinen und Generatoren verursacht, einerseits aufgrund der großen Mengen an Metallen (Stahl, Kupfer, Aluminium) und damit verbundenen Fertigungsprozessen andererseits auch durch den Einsatz von ,umweltschädlichen ' Stoffen (z. B. Epoxidharze). 
Abb. 3 Schematische Darstellung des Erntefaktors (Quelle: Wall 2011)

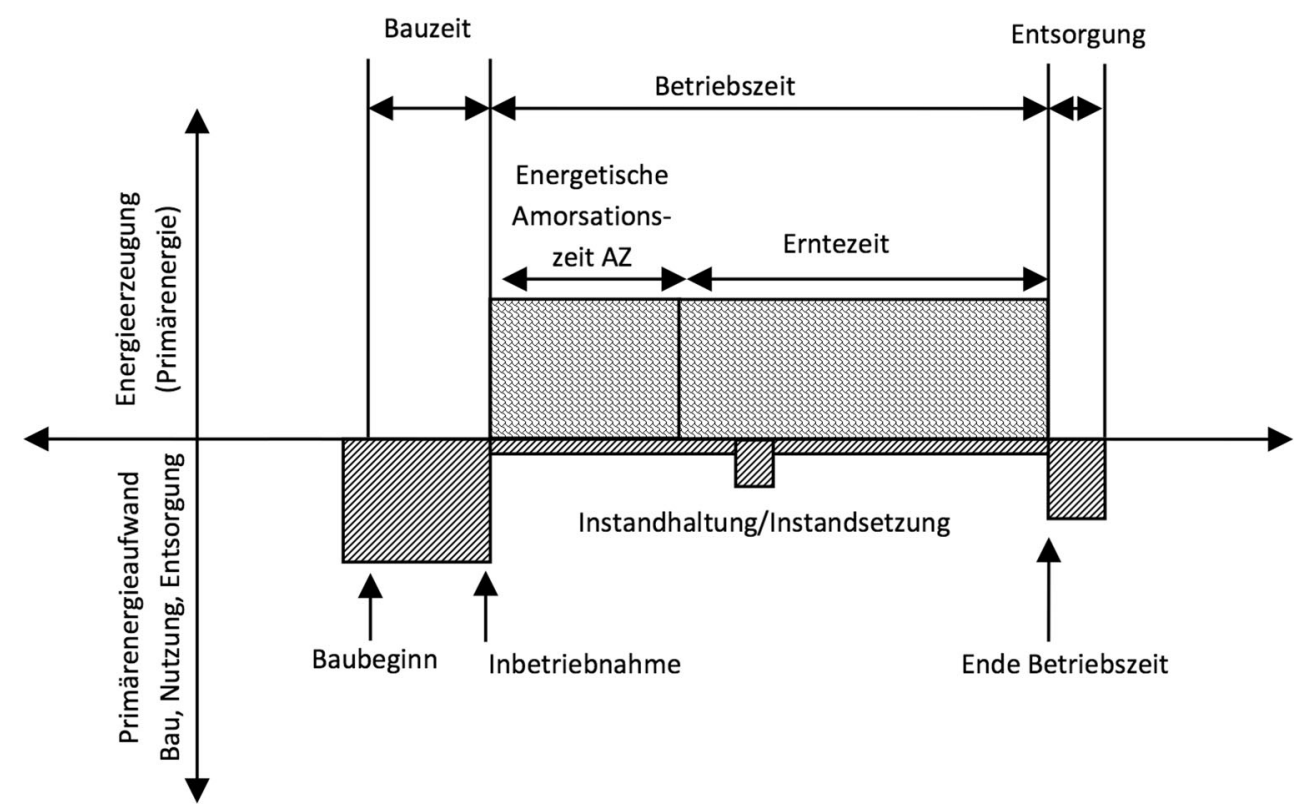

\subsection{Erntefaktor}

Die Berechnung der kumulierten Energieaufwendungen ermöglicht auch die Auswertung des „Erntefaktors“ des Wasserkraftwerks. Mit Hilfe dieses Erntefaktors kann eine Auskunft über die Energieeffizienz einer Energieerzeugungstechnologie gegeben werden. In Abb. 3 ist die Ermittlung des Erntefaktors grafisch dargestellt. Ein hoher Erntefaktor bedeutet eine gute energetische Performance, welche aber nicht zwingend eine ebenso gute ökologische Performance impliziert. Ist der Erntefaktor größer als 1, wird durch das System mehr Energie erzeugt, als für Herstellung, Betrieb und Rückbau desselbigen aufgewendet werden muss (auch als ,graue Energie“ bezeichnet).

Im Vergleich mit Literaturangaben gilt es besonders die Systemgrenzen und den betrachteten Lebenszyklus zu berücksichtigen, da diese wesentlich die damit verbundenen Energie- und Materialströme beeinflussen. Für das gegenständliche Kleinwasserkraftwerk wurde ein Erntefaktor von 25 berechnet, d. h. über den gesamten Lebenszyklus wird um das 25-fache mehr an Energie erzeugt, als für Herstellung, Betrieb und Rückbau notwendig ist. Dies entspricht in der Größenordnung der Bandbreite der einschlägigen Literatur (Kaltschmitt und Streicher 2009). Wobei bei größeren Wasserkraftwerksanlagen ein höherer Erntefaktor möglich ist, da die notwendigen Aufwendungen der Herstellung nicht direkt mit der erzeugten Energie korrelieren (Gagnon 2005).

\section{Diskussion}

Im Zuge der Arbeit konnte gezeigt werden, dass die Energieerzeugung mit Wasserkraft regenerativ ist. Jedoch sind für eine ganzheitliche Nachhaltigkeitsbetrachtung mehr Indikatoren $\mathrm{zu}$ berücksichtigen, der Erntefaktor alleine, als Abbildung der kumulierten Energieaufwendungen, erscheint als nicht ausreichend. Die Methodik der Ökobilanzierung wird ihrem Zweck der unterstützenden Entscheidungsfindung gerecht und kann einen Beitrag dazu leisten, verschiedene Energieerzeugungstechnologien miteinander zu vergleichen und Ansätze für eine Verwendung alternativer Materialen als auch Bauverfahren zu liefern. Wesentliche energetische Aufwendungen sind im gegenständlichen Beispiel auch auf die Zementerzeugung zurückzuführen. Anhand einer Sensitivitätsanalyse der verwendeten Datensätze für die Baustoffe (z. B. Beton, Stahl) können Ansätze für weitere baustofftechnologische Optimierungen abgeleitet werden (Fischer und Juhart 2014; Juhart et al. 2015). Diese Maßnahmen müssen konkret mess- und nachweisbar sein, um diese ökologischen Informationen kommunizieren zu können. Einen Ansatzpunkt stellen hier auch Umweltproduktdeklarationen (engl. Environmental Product Declarations EPD) dar, diese bilden die Datengrundlage für die ökologische Gebäudebewertung nach EN 15978 (2010). Umweltproduktdeklarationen basieren auf der internationalen Norm EN ISO 14025 (2011); ISO 14040 ff (2006) als auch auf der ÖNORM EN 15804 (2011) und sind geeignet, als Nachweis für die Umweltansprüche in der öffentlichen Beschaffung herangezogen zu werden. Zukünftig werden diese vermehrt in Bewertungssystemen für Gebäude oder Bauwerke zum Einsatz kommen (Passer et al. 2015). 
Auch für den Nachweis der umweltbezogenen Qualität von Strom sind vor allem produktspezifische Ökobilanzdaten entscheidend, da hier die Energie- und Stoffströme im Detail abgebildet werden können und dadurch die $\mathrm{Zu}$ sammensetzung des Strommixes klar ersichtlich dargestellt werden kann. Für Wasserkraftwerke gibt es diesbezüglich schon erste Deklarationen (Axpo AG 2007). Jedoch können mit Hilfe der Ökobilanz nicht alle ökologischen Aspekte adressiert werden, beispielsweise sind durch diese Berechnungen keine Rückschlüsse auf die ökologische Durchgängigkeit (z. B. Fischaufstiegshilfen) möglich.

\section{Ausblick - Zukünftige Anwendung im Bauwesen}

Wie in der gegenständlichen Berechnung des Erntefaktors eines Kleinwasserkraftwerks dargestellt, werden für die Quantifizierung von potentiellen Umweltwirkungen verlässliche und belastbare Informationen zu den Ausgangsdaten der Sachbilanz benötigt, um eine Aussage über die nachhaltige Performance eines Bauwerks zu liefern. Diese, für die Bewertung, notwendigen Informationen zu den Umweltwirkungen können anhand von Environmental Product Declarations (EPD) transparent kommuniziert werden. Der inhaltlichen Weiterentwicklung von einheitlichen und standardisierten EPDs folgend, bedarf es auch deren Verankerung in den Produktnormen und Zulassungen, erste Schritte in diese Richtung stellt die Grundanforderung Nr. 7 der Bauprodukteverordnung dar zur nachhaltigen Nutzung der natürlichen Ressourcen. Hier können EPDs als Produktnachweise dienen, dass Bauwerke diesen Vorgaben entsprechend entworfen, errichtet und rückgebaut werden können. EPDs werden von unabhängigen Dritten geprüft und qualitätsgesichert. In Österreich erfolgt dies durch die Bau-EPD GmbH. Diese verifizierten Umweltinformationen können auch als Nachweise für die jeweiligen Produkte im Zuge der Nachhaltigkeitsbewertung herangezogen werden und stellen hier ein geeignetes Informationsformat für die Evaluierung der ökologischen Qualitäten dar. Besonders in der Entwurfs- und Planungsphase ermöglichen diese standardisierten Umweltdaten einen groben Vergleich von verschiedenen Gebäudekonzepten hinsichtlich deren umweltbezogener Qualitäten. Des Weiteren können diese anschließend auch im umweltorientierten Beschaffungsvorgang (GPP) eingesetzt werden und später zur Dokumentation und Nachweisführung im Rahmen der ökologischen Baubegleitung verwendet werden.

Neben der Betrachtung der ökologischen Qualitäten von Bauprodukten bedarf es zukünftig noch mehr die entsprechenden Prozesse in der Entwurfs- und Planungsphase zu berücksichtigen. Dabei sind besonders die Wechselwirkungen zwischen der Produktperformance und der Gesamtbe- wertung des Bauwerks von Bedeutung. Die ökologischen Informationen sind hier wesentlich für die Zielerreichung hinsichtlich der grauen Energie sowie Ressourceneffizienz relevant, da diese Ziele nur durch eine optimierte Kombination der Produktionsfaktoren erreicht werden können.

Angesichts des fortschreitenden Klimawandels steht eine notwendige Minimierung von Treibhausgasen im Mittelpunkt. Dies ist nicht nur Ziel der Energiewirtschaft, sondern beeinflusst zusehends auch die Bauwirtschaft. Es bedarf einer ganzheitlichen Betrachtung der Projekte über den gesamten Produktzyklus (von der Wiege bis zur Bahre), um Effizienzpotenziale zu nutzen und Innovationen zu fördern. Für zukünftige Projekte können solche Untersuchungen auch hinsichtlich der verwendeten Bauprodukte und dem Nachweis der umweltbezogenen Qualität anhand von EPDs von zunehmender Relevanz sein. Im Zuge der öffentlichen Beschaffung stellt dabei die nachhaltigkeitsorientierte Ausschreibung und Vergabe von Bauleistungen einen Forschungsschwerpunkt dar (Wall 2015). Im Rahmen der Bearbeitung wird auch der Frage nachgegangen, wie kann eine Quantifizierung von Umweltwirkungen in Form einer Ökobilanzierung verstärkt im Beschaffungsvorgang berücksichtigt werden, um hier durch die besondere Betrachtung der Prozessqualitäten für eine Lebenszyklusoptimierung zu sorgen.

Danksagung Der Autor bedankt sich bei der Energie Steiermark AG für die zur Verfügung gestellten Projektunterlagen des Kleinwasserkraftwerks.

Open Access. This article is distributed under the terms of the Creative Commons Attribution 4.0 International License (http:// creativecommons.org/licenses/by/4.0/), which permits unrestricted use, distribution, and reproduction in any medium, provided you give appropriate credit to the original author(s) and the source, provide a link to the Creative Commons license, and indicate if changes were made.

\section{Literatur}

AXPO AG (2007) Umweltdeklaration Niederdruckkraftwerk WildeggBrugg. AXPO Hydro Energie, Baden

EN ISO 14025:2011 - Norm - Umweltkennzeichen und -deklarationen - Typ III Umweltdeklarationen Grundsätze und Verfahren (ISO 14025:2006)

Fischer GF, Juhart J (2014) Umweltwirkungen von Zusatzstoffen und Zusatzmitteln für ökologisch optimierten Beton. In: Nachhaltig Bauen mit Beton: Werkstoff und Konstruktion, S 231-236

Gagnon L (2005) Electricity Generation Options: Energy Payback Ratios: Life-cycle assessment of electricity generation options. http://tc4.iec.ch/FactSheetPayback.pdf. Zugegriffen: 24. Nov. 2015

Giegrich et al (2014) Ökologische Rentabilität der Metallrückgewinnung - Grenzbetrachtungen bei metallhaltigen mineralischen Abfällen. uwf UmweltWirtschaftsorum 22(2):187-194. doi:10.1007/s00550-014-0328-1

Giesecke et al (2014) Wasserkraftanlagen - Planung, Bau und Betrieb. Springer, Berlin Heidelberg, S 20-23 
Juhart et al (2015) Ökologische Optimierung von Beton mit inerten Mikro- und Eco-Füllern. In: Tagungsbericht 19. Internationale Baustofftagung Ibausil Weimar., S 1073-1084

ÖNORM EN ISO 14040:2006 - Norm - Umweltmanagement - Ökobilanz Grundsätze und Rahmenbedingungen

ÖNORM EN 15804:2011 - Norm - Nachhaltigkeit von Bauwerken Umweltproduktdeklarationen für Produkte - Grundregeln für die Produktkategorie Bauprodukte

ÖNORM EN 15978:2010 - Norm - Nachhaltigkeit von Bauwerken Bestimmung der Umweltleistung von Gebäuden - Berechnungsmethode

Passer et al (2015) Environmental Product Declarations entering the building sector - critical reflections based on 5 years experience in different European countries. Int J Life Cycle Assess 20(9):1199-1212. doi:10.1007/s11367-015-0926-3

Pöyry Energy (2008) Wasserkraftpotenzialstudie Österreich. Endbericht. Pöyry Energy GmbH im Auftrag des VEÖ. Verband der Elektrizitätsunternehmen Österreichs, Wien

Suwanit W, Gheewala S (2011) Life cycle assessment of mini-hydropower plants in Thailand. Int J Life Cycle Assess 16(9):849-858. doi:10.1007/s11367-011-0311-9

Kaltschmitt M, Streicher W (2009) Regenerative Energien in Österreich. Vieweg \& Teubner, Wiesbaden

Unger J, Hurtado A (2014) Energetische Beurteilungskriterien. In: Alternative Energietechnik. Springer Fachmedien, Wiesbaden, S 81-87

Vega et al (2013) Life cycle inventory of construction of Hydroelectric dam in Chile. In: Proceedings of the 6th International Conference on Life Cycle Management in Gothenburg 2013. http://
conferences.chalmers.se/index.php/LCM/LCM2013/paper/view/ 602/203. Zugegriffen: 24. Nov. 2015

Wall J (2011) Erntefaktor in der Wasserkraft. Masterarbeit, Technische Universität Graz

Wall J (2015) Ausschreibung und Vergabe hinsichtlich der derzeitigen Berücksichtigung von nachhaltigen Aspekten. In: Achammer CM, Kovacic I (Hrsg) Integrale Planung für Industrie-Bau 4.0 Praxisreport 2015, Institut für Interdisziplinäres Bauprozessmanagement. TU Wien, Klein Publishing GmbH, Wien, S 114-123

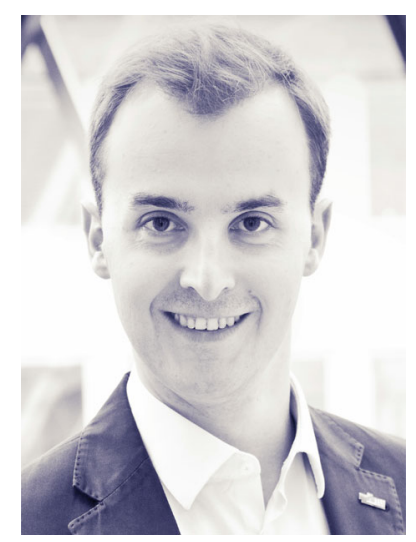

Johannes Wall 